http://jmscr.igmpublication.org/home/ ISSN (e)-2347-176x ISSN (p) 2455-0450 crossref DOI: https://dx.doi.org/10.18535/jmscr/v7i7.124

\title{
Ante Mullerian Hormone versus Antral Follicle Count as a predictor of ovarian response to controlled ovarian hyper stimulation in Assisted Reproductive Technique- A prospective study
}

\author{
Authors \\ Parvathy $\mathbf{T}^{1}$, Fessy Louis $\mathbf{T}^{2^{*}}$, Ramesh.P ${ }^{1}$, Aparna $\mathbf{N}^{1}$, Avani Pillai ${ }^{3}$, Jayashree $\mathbf{N}^{4}$, \\ Sundaram $\mathrm{KR}^{\mathbf{5}}$, Renjitha $\mathbf{N}^{\mathbf{6}}$ \\ ${ }^{1}$ Senior resident, Dept of Reproductive Medicine and Surgery \\ ${ }^{2}$ Assoc. Prof, Dept of Reproductive Medicine and Surgery \\ ${ }^{3}$ Asst Prof, Dept of Reproductive Medicine and Surgery \\ ${ }^{4}$ Professor and Head, Dept of Reproductive Medicine and Surgery \\ ${ }^{5}$ Professor and Head, Dept of Biostatistics, ${ }^{6}$ Lecturer, Dept of Biostatistics \\ Amrita Institute of Medical Sciences, Amrita Viswa Vidyapeetham University, India \\ *Corresponding Author \\ Dr Fessy Louis T \\ Assoc. Prof, Dept of Reproductive Medicine and Surgery, Amrita Institute of Medical Sciences, Amrita \\ Viswa Vidyapeetham University, India
}

\begin{abstract}
Background: Ovarian reserve assessment in Assisted Reproductive techniques (ART) and the decision based on its value is a diagnostic dilemma. Among the tests available to assess ovarian reserve, the most commonly used tests include FSH, AFC and AMH.

Objectives: To assess whether AMH or AFC is a better predictor of ovarian response (as evidenced by folliculogenesis) and cycle outcome in ART and also to see the correlation between AMH and AFC.

Material and Methods: This was a prospective comparative study conducted in the Department of Reproductive Medicine and Surgery at Amrita Fertility Centre, Kochi from July 2017 to September 2018. The study group comprised of 80 women recruited for ICSI. The number of follicles more than $12 \mathrm{~mm}$ on day of trigger was the primary outcome studied. After trigger and oocyte retrieval followed by ICSI the rest of the dependent parameters of $\mathrm{COH}$ were assessed including the pregnancy rate. Based on the AMH and AFC subgroups (low / normal/ high), the various parameters were compared. Results were analyzed using SPSS version 20.
\end{abstract}

Results: Eighty women who underwent $\mathrm{COH}$ and ICSI were followed up till 12 weeks of pregnancy. Patients divided into AMH and AFC low, normal and high groups. With respect to the follicle number, on comparing between the AMH and AFC groups there was no difference between the low, normal and high sub groups ( $p$ $=0.76$ ).

Conclusion: $A M H$ and $A F C$ are having same ability and clinical value in prediction of ovarian response. So AFC may be considered as a substitute for the expensive AMH estimation in predicting the ovarian response to stimulation.

Keywords: Ante Mullerian Hormone, Antral Follicle Count, Controlled Ovarian Hyper stimulation, Follicle number, Pregnancy. 


\section{Introduction}

One of the most important determinant factor for infertility as far as female is concerned is the age. With advancing female age there is reduction in female's fecundity due to reduction in both the quantity and quality of the oocytes.

The term "ovarian reserve" has been used to describe a woman's reproductive potential, specifically, the number and quality of oocytes she possesses ${ }^{1}$. Women of similar age may express large difference in their ovarian reserve. Thus it is a great challenge to design an ovarian reserve test that estimate an individual's reproductive life span at a given age ${ }^{2}$.

A number of methods have now been described, all intended to predict fertility or to provide prognostic information regarding the likelihood of successful treatment in infertile women. The ovarian reserve tests help in predicting the ovarian response and also for individualization of treatment protocol for getting the optimal response. Lot of ovarian reserve tests are currently available. They include both biochemical static and provocative tests and ultrasound imaging of the ovaries. Among them the most commonly used tests include FSH, AFC and AMH.

There are a lot of studies available with respect to $\mathrm{AMH}$ and AFC as ovarian reserve tests. Most of the studies are from western world which cannot be extrapolated to our population. Also, there is no clear cut evidence that one is superior over the other. We aimed to assess whether AMH or AFC is a better predictor of the folliculogenesis as described by ovarian response.

\section{Methods}

This was a prospective comparative study involving 80 women presented at Amrita Fertility Centre, Kochi, who underwent Intra Cytoplasmic sperm injection (ICSI) from July 2017 to September 2018. Based on mean and SD of number of follicles reported in the low and normal group of ovarian reserve, observed in an earlier publication ${ }^{3}$ and with $95 \%$ confidence and $90 \%$ power, minimum sample size came to be about 5 in each group. Since the proportion of normal and low was 2:1, minimum sample size with $90 \%$ confidence and $90 \%$ power, came to 15 and 10 in normal and low group respectively. Since the proportion of high could be taken almost equal to normal group (from experience), we planned to include 15 cases in high group also. Approval was obtained from the Dissertation review committee and the Institutional ethics committee (Amrita Institute of Medical Sciences, Kochi). Written informed consent was obtained from all the participants.

In all the recruited subjects, detailed history with respect to nature, duration, etiology of infertility, co- existing medical illness and history of previous surgeries, if any, were obtained.

Based on the AMH results women were classified into 3 groups as High OR $(>4 \mathrm{ng} / \mathrm{ml})$, Normal OR (1-4ng/ml), Low OR $(<1 \mathrm{ng} / \mathrm{ml})$ and similarly, based on AFC results, classified as High OR (>12), Normal OR (7-12) and poor OR (<7).

$\mathrm{AFC}, \mathrm{AMH}$ and other hormonal assay were measured on day 2-4 of spontaneous or induced cycles. Then the cases were subjected to long protocol of stimulation. The subjects were serially followed up with ultrasound and hormone level after starting stimulation with gonadotropins. The number of follicles more than $12 \mathrm{~mm}$ on day of trigger was the primary outcome studied. After trigger and oocyte retrieval followed by ICSI the rest of the parameters of the study assessed including oocyte number, embryo number, pregnancy rate, clinical pregnancy rate and the ongoing pregnancy rate. Based on the $\mathrm{AMH}$ and AFC subgroups the various parameters were compared to predict which among AMH or AFC was better.

Statistical Analysis was done using IBM SPSS statistics 20 windows (SPSS Inc., Chicago, USA). For all the continuous variables the results are given in mean \pm standard deviation and for categorical variables as frequency and percentage. To test the statistical significance of the mean difference of the continuous variables between $\mathrm{AMH}$ and $\mathrm{AFC}$ in different categories, paired t-test was applied. To study the correlation between AMH and AFC, 
Pearson correlation coefficient was computed. To test the statistical significance of the difference with respect to categorical variables between $\mathrm{AMH}$ and AFC sub groups, McNemar's Chi square test was used.

\section{Results}

Mean age of the patients in the study group was $32.74 \pm 4.9$ years.

The basal characteristics are depicted in Table 1 .

The majority $(>50 \%)$ were in the normal BMI group. Chief cause of infertility was female factor $(58.75 \%)$ and the majority $(56.3 \%)$ had duration of infertility between 5- 10 years.

In the study population, $50 \%$ had more than 10 oocytes on stimulation. The number of oocytes was between 5 to 10 in $33.75 \%$ of cases and the remaining $16.25 \%$ had less than 5 oocytes on stimulation.

Out of the 80 subjects, $42.5 \%$ had biochemical pregnancy. Clinical pregnancy was observed in $41.25 \%$.

Table 1: Basal characteristics of the Population. $(\mathrm{n}=80)$

\begin{tabular}{|l|c|c|}
\hline Parameter & Category & Percentage \\
\hline \multirow{3}{*}{$\begin{array}{l}\text { Socio economic } \\
\text { status }\end{array}$} & Low & 03.75 \\
\cline { 2 - 3 } & Middle & 46.25 \\
\cline { 2 - 3 } & High & 50.00 \\
\hline Cause of Infertility & Female factor & $58.75 \%$ \\
\cline { 2 - 3 } & Male Factor & $16.5 \%$ \\
\cline { 2 - 3 } & Combined & $5 \%$ \\
\cline { 2 - 3 } & Unexplained & $16.25 \%$ \\
\hline Follicle number & $<5$ & $12.5 \%$ \\
\cline { 2 - 3 } & $5-10$ & $40 \%$ \\
\cline { 2 - 3 } & $>10$ & $47.5 \%$ \\
\hline BMI $\left(\mathrm{kg} / \mathrm{m}^{2}\right)$ & $<18$ & 08.80 \\
\cline { 2 - 3 } & $18-25$ & 51.30 \\
\cline { 2 - 3 } & $>25$ & 40.00 \\
\hline \multirow{3}{*}{$\begin{array}{l}\text { Duration } \\
\text { Infertility }\end{array}$} & $<5$ years & 28.80 \\
\cline { 2 - 3 } & $5-10$ years & 56.30 \\
\cline { 2 - 3 } & $>10$ years & 15.00 \\
\hline
\end{tabular}

The number of patients in the high, normal and low groups of AMH and AFC are depicted in Table 2. With respect to the follicle number on comparing between the AMH and AFC groups there was no difference between the low, normal and high sub groups (Table 3 and Figure 1).
Table 2- Number of subjects distributed across Low mid and High groups of AMH and AFC

\begin{tabular}{|l|c|c|c|}
\hline Parameter & AMH & AFC & Correlation (r) \\
\cline { 1 - 3 } Low & $25(31.25 \%)$ & $29(36.25 \%)$ & \multirow{2}{*}{} \\
\cline { 1 - 2 } Mid & $38(47.5 \%)$ & $27(33.75 \%)$ & 0.44 \\
\cline { 1 - 2 } High & $17(21.25 \%)$ & $24(30 \%)$ & $\mathrm{p}<0.001$ \\
\hline Total & $80(100 \%)$ & $80(100 \%)$ & \\
\hline
\end{tabular}

Table - 3 Comparison of average follicle number among AMH and AFC groups

\begin{tabular}{|l|c|c|c|}
\hline Sub group & $\begin{array}{c}\text { AFC(mean } \pm \\
\text { SD) }\end{array}$ & $\begin{array}{c}\text { AMH(mean } \pm \\
\text { SD })\end{array}$ & $p$ value \\
\hline Low & $07.25 \pm 4.82$ & $6.76 \pm 5.34$ & 0.54 \\
\hline Normal & $09.48 \pm 3.59$ & $9.58 \pm 3.91$ & 0.99 \\
\hline High & $15.55 \pm 5.46$ & $16.08 \pm 5.23$ & 0.76 \\
\hline
\end{tabular}

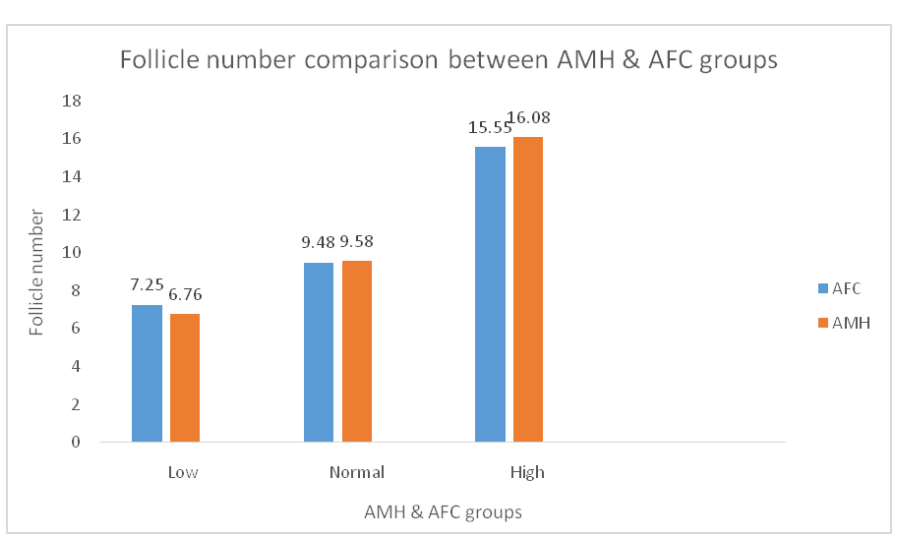

Figure 1: Comparison of the mean follicle number obtained in the low, normal and high groups of AFC and $\mathrm{AMH}$

\section{Discussion}

The prediction of ovarian response as evidenced by the folliculogenesis is not significantly different between the tests of ovarian reserve AMH and AFC. One of the most important causes for female factor infertility is ovulatory dysfunction. Large number of tests are now available to determine the ovarian reserve. Among them the most important ones include the female age, baseline FSH, AMH and AFC. In the present study we tried to compare the effectiveness of $\mathrm{AMH}$ and $\mathrm{AFC}$ as a predictor of ovarian response to $\mathrm{COH}$ in ART.

The mean age of subjects in the study group were $32.74 \pm 4.9$ years. The cycle details of the present study include that about $47.5 \%$ cases were having follicle number more than 10 on stimulation, $40 \%$ had follicle number between 5 to 10 and $12.5 \%$ cases had follicle number less than 5 after $\mathrm{COH}$. 
The oocytes retrieved were more than 10 in $50 \%$ cases, $33.75 \%$ cases oocyte between 5 to 10 and $16.25 \%$ cases had less than 5 oocytes retrieved after stimulation. Out of the 80 cases $42.5 \%$ and $41.25 \%$ were the positive cases in the biochemical pregnancy, clinical pregnancy.

Table 3 shows the comparison of follicle number among the AMH and AFC subgroups. On comparing between the $\mathrm{AMH}$ and AFC groups there was no difference in the follicle number between the groups. From the study of Lee et al, similar conclusion was drawn that those with higher AMH and AFC values, there were higher number of follicles and oocyte retrieved on stimulation and there was no statistical difference between the groups ${ }^{4}$.

Various studies ${ }^{(5-7)}$ are in concordance with our findings. Hence it is prudent to conclude that $\mathrm{AMH}$ and AFC are not superior over each other in predicting ovarian response as evidenced by folliculogenesis following ART.

In the present study the correlation of AFC with $\mathrm{AMH}$ was found to be 0.44 which was statistically significant on comparing between the high, normal and low group and was statistically significant ( $p$ value $=<0.001)$. (Table 2$)$

The categories of AMH and AFC were arbitrarily chosen based on experience and the validity of the classification of low, normal and high was not accurately established. The groups were not homogenously distributed between the low, normal and high categories. Inclusion of other protocols would have improved the generalizability of the results.

In summary, the present study has shown that AMH and AFC are having same level of accuracy and clinical value in prediction of ovarian response. So AFC can be substituted for AMH in pre IVF ovarian assessment as the predictive ability of both $\mathrm{AMH}$ and AFC for COS outcome are almost comparable from our study.

\section{Conclusion}

$\mathrm{AMH}$ and AFC are having the same level of accuracy and clinical value in prediction of ovarian response. So AFC can be considered as a substitute of expensive $\mathrm{AMH}$ estimation in predicting the $\mathrm{COH}$ outcome.

\section{Conflicts of Interest: Nil reported}

\section{References}

1. Practice Committee of the American Society for Reproductive Medicine. Testing and interpreting measures of ovarian reserve: a committee opinion. Fertil Steril 2015;103: e917.

2. Broer SL, Mol BW, Broekmans FJ. The role of antimullerian hormone in prediction of outcome after IVF: comparison with antral follicle count. Fertil Steril. 2009; 91:705 -14.

3. Himabindu, Y., Sriharibabu, M., Gopinathan, K., Satish, U., Louis, T.F., Gopinath, P. Antimullerian hormone and antral follicle count as predictors of ovarian response in assisted reproduction. J. Hum. Reprod 2013; Sci. 6, $27-$ 31.

4. Li, H.W., Lee, V.C., Lau, E.Y., Yeung, W.S., Ho, P.C., Ng, E.H. Role of baseline antral follicle count and anti-Mullerian hormone in prediction of cumulative live birth in the first in vitro fertilization cycle: a retrospective cohort analysis. PLoS One 8; 2013: e61095.

5. La Marca A, Sighinolfi G, Radi D. Antimullerian hormone (AMH) as a predictive marker in assisted reproductive technology (ART). Hum Reprod Update. 2010; 16:113-30.

6. Lukaszuk, K., Kunicki, M., Liss, J., Lukaszuk, M., Jakiel, G. Use of ovarian reserve parameters for predicting live births in women undergoing in vitro fertilization. Eur. J. Obstet. Gynecol. Reprod. Biol 2013; 168, 173-177.

7. Polyzos, N.P., Tournaye, H., Guzman, L., Camus, M., Nelson, S.M. Predictors of ovarian response in women treated with corifollitropinalfa for in vitro fertilization/ intracytoplasmic sperm injection. Fertil. Steril 2013; 100, 430-437. 